\title{
Quantum mechanics in the brain
}

\section{Does the enormous computing power of neurons mean consciousness can be explained within a purely neurobiological framework, or is there scope for quantum computation in the brain?}

\section{Christof Koch and Klaus Hepp}

The relation between quantum mechanics and higher brain functions, including consciousness, is often discussed, but is far from being understood. Physicists, ignorant of modern neurobiology, are tempted to assume a formal or even dualistic view of the mind-brain problem. Meanwhile, cognitive neuroscientists and neurobiologists consider the quantum world to be irrelevant to their concerns and therefore do not attempt to understand its concepts. What can we confidently state about the current relationship between these two fields of scientific inquiry?

All biological organisms must obey the laws of physics, both classical and quantum. In contrast to classical physics, quantum mechanics is fundamentally indeterministic. It explains a range of phenomena that cannot be understood within a classical context: the fact that light or any small particle can behave like a wave or particle depending on the experimental setup (wave-particle duality); the inability to simultaneously determine, with perfect accuracy, both the position and momentum of an object (Heisenberg's uncertainty principle); and the fact that the quantum states of multiple objects, such as two coupled electrons, may be highly correlated even though the objects are spatially separated, thus violating our intuitions about locality (entanglement).

Major philosophical and conceptual problems surround the process of making measurements in quantum mechanics. To illuminate the paradoxical nature of superposition - that is, the fact that particles or quantum bits (qubits) are allowed to exist in a superposition of states - Schrödinger proposed a celebrated thought experiment: a sealed box containing the quantum superposition of both a dead and a live cat. When an observer peers inside the box, measuring its content, the wave function, which describes the probability that the system will be found in any one particular state, is said to collapse, and the system will be found in one or the other state with known probability.

The role of the conscious observer in this measuring process has been hotly debated since the early days of quantum mechanics. It is fair to say, however, that consciousness has been only a place holder in a chain of mathematical formulae, without much relevance to the study of neural circuits in

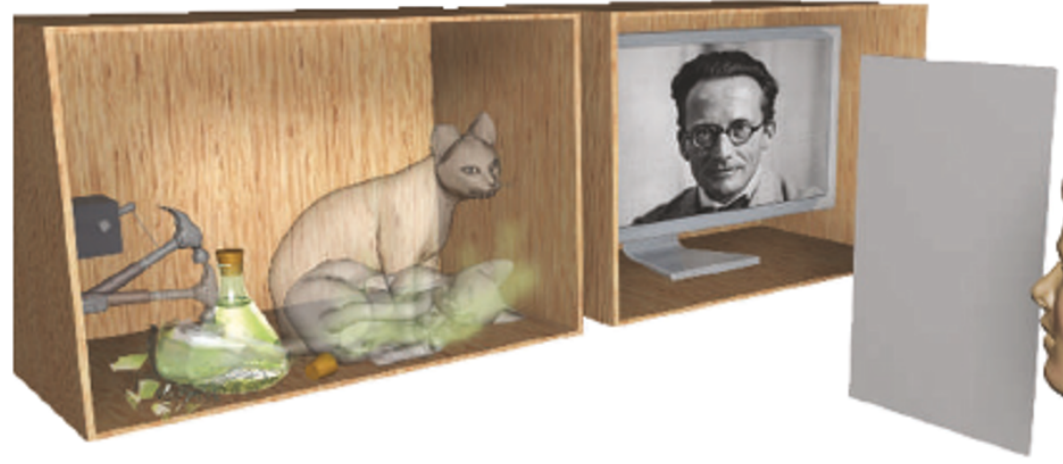

A thought experiment involving an observer looking at a super imposed quantum system with one eye, and at a succession of faces with the other, challenges the idea that a quantum framework is needed to explain consciousness.

intact organisms. Most quantum physicists view the brain as a classical instrument.

The critical question we are concerned with here is whether any components of the nervous system - a 300-degrees Kelvin tissue strongly coupled to its environment display macroscopic quantum behaviours, such as quantum entanglement, that are key to the brain's function.

Specific molecular machines and proteins have been proposed to implement quantum computations. The best known of such proposals is Penrose and Hameroff's hypothesis that the tubulin components of microtubules, filamentous protein polymers that form the cytoskeleton of cells, implement quantum computations.

\section{Lessons from quantum computers}

When kings build, their followers team up in international journals and conferences, confusing the general public about the distinction between science and poetry. But large quantum systems are notoriously difficult to analyse rigorously, except in highly idealized models or limits. Estimates based on the same unrealistic one-particle model, applied to trillions of interacting particles, show discrepancies of ten orders of magnitude in the work of different authors. It is therefore better to turn to hard experimental realities and abstract computational theory to find the neural correlates of quantum processes in the brain.

Quantum computations are difficult to implement. In its simplest version, a quantum computer transforms the state of many two-dimensional qubits using a reversible, linear, probability-conserving mapping via a sequence of externally controllable quantum gates into a final state with a probabilistic outcome. Quantum computation seeks to exploit the parallelism inherent in entanglement by assuring that the system is very likely to converge on the computationally desirable result.

This requires that the qubits are well isolated from the rest of the system. Coupling the system to the external world is necessary for the preparation of the initial state (the input); for the control of its evolution; and for the actual measurement (the output). However, all these operations introduce 'noise' into the computation (decoherence). Although some decoherence can be compensated for by redundancy and other faulttolerant techniques, too much is fatal.

In spite of an intensive search by many laboratories, no scalable large quantum computers are known. The record for quantum computation is the factoring of the number 15 by liquid-state nuclear magnetic resonance (NMR) techniques. Qubits and a set of universal quantum gates have been proposed in many different implementations, but all solutions have serious drawbacks: photons interact only weakly with one another; nuclear spins in individual molecules are few in number in current devices, as are trapped atoms or ions. This paints a desolate picture for quantum computation inside the wet and warm brain.

Although brains obey quantum mechanics, they do not seem to exploit any of its special features. Molecular machines, such as the light-amplifying components of photoreceptors, pre- and post-synaptic receptors and the voltage- and ligand-gated channel proteins that span cellular membranes and underpin neuronal excitability, are so large that they can be treated as classical objects. (Their relative molecular masses range from 20,000 to 200,000 ; the two main 
dimers of tubulin are around 55,000 .)

Two key biophysical operations underlie information processing in the brain: chemical transmission across the synaptic cleft, and the generation of action potentials. These both involve thousands of ions and neurotransmitter molecules, coupled by diffusion or by the membrane potential that extends across

\section{"Many previously mysterious aspects of perception are explainable in terms of} neuronal processing." are much more powerful than conventional algorithms (based on classical physics), are implemented in the nervous system. The most famous of these is Shor's procedure for factoring large integers for data encryption. However, in the past decade no quantum algorithm of similar power and applicability to Shor's has been found. And factortens of micrometres. Both processes will destroy any coherent quantum states. Thus, spiking neurons can only receive and send classical, rather than quantum, information. It follows that a neuron either spikes at a particular point in time or it does not, but is not in a superposition of spike and nonspike states.

The power of quantum mechanics is often invoked for problems that brains solve efficiently. Computational neuroscience is a young field and theories of complex neural systems, with all the variability of living matter, will never reach the precision of physical laws of well-isolated simple systems. It has already been demonstrated, however, that many previously mysterious aspects of perception and action are explainable in terms of conventional neuronal processing.

Two examples are models for the rapid recognition of objects (for example, animals or faces) in natural scenes, with performance approaching that of human observers, and the attentional selection of objects in cluttered images. The necessary mathematical operations - such as changes in synaptic weights, evaluating the inner product between presynaptic activity and synaptic weight, multiplication and stationary nonlinearities - are available to neurons. Indeed, there is an embarras de richesse of computational primitives implemented by synapses, dendrites and neurons. That is not to suggest that we understand how brains compute. But so far, there seems to be no need for quantum skyhooks.

The reason for the unprecedented computational power of nervous systems is their high degree of parallelism. For instance, filter-like operations in retinal or cortical cells in the visual stream are performed simultaneously on an entire image and thus are not limited by the tyranny of a single processor. Furthermore, unlike the von Neumann architecture of the programmable digital computer, the brain intermixes memory elements in the form of modifiable interconnections within the computational substrate, the neuronal membrane. Thus, no separate memory 'fetch' and 'store' cycles are necessary.

Much of the hope that quantum mechanics works in the brain is pinned to the supposition that quantum algorithms, which ing large numbers is not something for which the brain has much use.

Why should evolution have turned to quantum computation, so fickle and capricious, if classical neural-network computations are evidently entirely sufficient to deal with the problems encountered by nervous systems?

\section{Food for thought}

At this point, intrepid students of the mind point to qualia, the constitutive elements of consciousness. The subjective feelings associated with the redness of red or the painfulness of a toothache are two distinct qualia. As long as it remains mysterious how the physical world gives rise to such sensations, could one of the more flamboyant interpretations of quantum mechanics explain consciousness? Most provocatively, Roger Penrose has claimed that brains can evaluate noncomputable functions; that this ability is related to consciousness; that both this ability and consciousness require a yet-tobe-discovered theory of quantum gravity and that microtubules are the sites of the associated quantum gates.

The problem of consciousness and its neuronal correlates is beginning to emerge in outlines. The content of consciousness is rich and highly differentiated. It is associated with the firing activity of a very large number of neurons spread all over the corthalamus. Thus, any one conscious percept or thought must be expressed in a wideflung coalition of neurons firing together. Even if quantum gates exist within the confines of neurons, it remains totally nebulous how information of relevance to the organism would get to these quantum gates. Moreover, how would it be kept coherent across the milli- and centimetres separating individual neurons when synaptic and spiking processes, the primary means of neuronal communication on the perceptual timescale, destroy quantum information?

It is far more likely that the material basis of consciousness can be understood within a purely neurobiological framework, without invoking any quantum-mechanical deus ex machina.

We challenge those who call upon consciousness to carry the burden of the measurement process in quantum mechanics tex and associated satellites, such as the with the following thought experiment. Visual psychology has caught up with magicians and has devised numerous techniques for making things disappear. For instance, if one eye of a subject receives a stream of highly salient images, a constant image projected into the other eye is only seen infrequently. Such perceptual suppression can be exploited to study whether consciousness is strictly necessary to the collapse of the wave function.

Say an observer is looking at a superimposed quantum system, such as Schrödinger's box with the live and dead cat, with one eye while his other eye sees a succession of faces (see figure). Under the appropriate circumstances, the subject is only conscious of the rapidly changing faces, while the cat in the box remains invisible to him. What happens to the cat? The conventional prediction would be that as soon as the photons from this quantum system encounter a classical object, such as the retina of the observer, quantum superposition is lost and the cat is either dead or alive.

This is true no matter whether the observer consciously saw the cat in the box or not. If, however, consciousness is truly necessary to resolve the measurement problem, the animal's fate would remain undecided until that point in time when the cat in the box becomes perceptually dominant to the observer. This seems unlikely but could, at least in principle, be empirically verified.

The empirical demonstration of slowly decoherent and controllable quantum bits in neurons connected by electrical or chemical synapses, or the discovery of an efficient quantum algorithm for computations performed by the brain, would do much to bring these speculations from the 'far-out' to the mere 'very unlikely'. Until such progress has been made, there is little reason to appeal to quantum mechanics to explain higher brain functions, including consciousness.

Christof Koch is in the Division of Biology and the Division of Engineer ing and Applied Science, 216-76, California Ins titute of Technology, Pasadena, California 91125, USA. Klaus Hepp and Christof Koch are at the Institute of Neuroinformatics, the University of Zürich and ETH, Zürich, Switzerland.

FURTHER READING

Hepp, K in Quantum Future: Lecture Notes in Physics (eds Blanchard, P. \& Jad czyk, A.) 517, 92-104 (1998). Koch, C. Biophysics of Computation: Information Processing in Single Neurons (OxfordUniv. Press, New York, 1999).

Koch, C. The Quest for Consciousness: A

Neurobiological Approach (Ro berts, Colorado, 2004) Nielsen, M.\& Chuang, I. Quantum Computation and Quantum Information (Cambridge Univ. Press, Cambridge, 2002).

Penrose, R. TheEmperor's New Mind (Oxford Univ. Press, Oxford 1989). 